\title{
Interest Point Detectors for Visual SLAM
}

\author{
Óscar Martínez Mozos ${ }^{1}$, Arturo Gil $^{2}$, Monica Ballesta ${ }^{2}$, and Oscar Reinoso ${ }^{2}$ \\ 1 Department of Computer Science, University of Freiburg, Germany \\ 2 Department of Systems Engineering, Miguel Hernández University, Spain
}

\begin{abstract}
In this paper we present several interest points detectors and we analyze their suitability when used as landmark extractors for vision-based simultaneous localization and mapping (vSLAM). For this purpose, we evaluate the detectors according to their repeatability under changes in viewpoint and scale. These are the desired requirements for visual landmarks. Several experiments were carried out using sequence of images captured with high precision. The sequences represent planar objects as well as $3 \mathrm{D}$ scenes.
\end{abstract}

\section{Introduction}

Acquiring maps of the environment is a fundamental task for autonomous mobile robots, since the maps are required in different higher level tasks. As a result, the problem of simultaneous localization and mapping (SLAM) has received significant attention. Typical approaches use range sensors to build maps in two and three dimensions (see, for example, [1-3] [4-6]). In recent years there is an increasing interest on using cameras as sensors. Such approach is sometimes denoted as visual SLAM (vSLAM). Cameras offer higher amount of information and are less expensive than lasers. Moreover, they can provide 3D information when stereo systems are used.

Usual approaches using vision apply a feature-based SLAM, in which visual features are used as landmarks. The main issue when using vSLAM is how select suitable features on the images to be used as reliable landmarks. When the map to construct has three dimensions, the landmarks must additionally be robust to changes in the scale and viewpoint. Different vision features has been used for mapping and localization using monocular or stereo vision, as for example, lines [7], region of interest [8]; and interest points, as SIFT [9-11], Harris corner detector $[12,13]$ or SURF [14]. The interest points detectors have received most of the attention in vSLAM. The points detected are typically invariant under rotation, translation, scale and only partially invariant under changes in viewpoint. These theoretical properties made them suitable for been used as visual landmarks. In practice, however, the stability of the points is not always maintained and the matching between them becomes difficult. Some solutions have been proposed to solve this problem, as combining several methods in one detector [15] or tracking the points during several frames to keep the stability [16, 10]. However, the question of which interest point detector is more suitable for vSLAM is still open. 
In this paper we present several evaluations of different point detectors that are typically used in vSLAM. The extracted points used as landmarks should be robust under scale and viewpoint changes. These requirements are necessary for vSLAM, since the robot must be able to detect and associate new landmarks to previous ones. Under these conditions we analyze the repeatability of the points in consecutive images and the probability of been detected in future ones.

The rest of the paper is organized as follows. After discussing some related work in Section 2, we present different interest point detectors in Section 3. Section 4 introduces the evaluation methods used in this work. Several experiments are presented in Section 5. We finally conclude in Section 6.

\section{Related Work}

Visual SLAM has been an interesting topic in mobile robotics for the last years. Different methods has been used to extract visual landmarks. Lemaire and Lacroix [7] use segments as landmarks together with and EKF-based SLAM approach. Frintrop et al. [8] extract regions of interest (ROI) using the attentional system VOCUS. Other authors use SIFT features as landmarks in the 3D space [9, 16]. Little et al. [17] and Gil et al. [10] additionally track the SIFT features to keep the most robust ones; and Valls Miro et al. [11] use SIFT to map large environments. Harris corner detectors has also been used as landmarks for monocular SLAM (Davison and Murray [12]) or in Autonomous Blimps (Hygounenc et al. [13]). Finally, Murillo et al. [14] present a localization method using SURF features.

In the context of matching and recognition, many authors have presented their works evaluating several interest point detectors. The work presented by Mikolajczyk and Schmid [18], uses different detectors to extract affine invariant regions, but only focuses on the comparison of different description methods. In [19], a collection of detectors is evaluated. The criteria used measures the quality of these features for tasks like image matching, object recognition and $3 \mathrm{D}$ reconstruction. However they do not take into account the repeatability in the successive frames of a sequence. In contrast to the previous works we evaluate the different interest point detectors under the particular conditions of vSLAM.

\section{Interest Point Detectors}

Along this paper we suppose that a mobile robot is used for constructing the map of the environment. The robot is equipped with a camera used to acquire images. Interest points are then extracted from these images and used as landmarks. We also suppose that the height of the camera on the robot is fixed as well as its orientation. This is the typical configuration in visual SLAM systems. Additionally, we assume that visual landmarks are static, i.e. they do not change their position or oriention during the experiments. According to the previous criterion, we following present five different interest point detectors used to extract visual landmarks. 


\subsection{Harris Corner Detector}

The Harris Corner Detector [20] is probably the most widely interest point detector used due to its strong invariance to scale, rotation and illumination variations, as well as image noise. The detector is based on the matrix $C(x, y)$ which is computed over a $p \times p$ patch for each interest point at position $(x, y)$ as:

$$
C(x, y)=\left(\begin{array}{cc}
\sum I_{x}^{2} & \sum I_{x} I_{y} \\
\sum I_{x} I_{y} & \sum I_{y}^{2}
\end{array}\right),
$$

where $I_{x}, I_{y}$ are the image gradients in horizontal and vertical direction. Let $\lambda_{1}$ and $\lambda_{2}$ be the eigenvalues of the matrix $C(x, y)$, we define the auto-correlation function $R$ as:

$$
R=\lambda_{1} \lambda_{2}-k\left(\lambda_{1}+\lambda_{2}\right)^{2} .
$$

This function will be sharply peaked if both of the eigenvalues are high. This means that shifts in any direction will produce a significant increase, indicating that it is a corner. A typical value for $k$ is 0.04 [12].

\subsection{Harris-Laplace}

The interest points extracted by the Harris-Laplace detector [21] are invariant to rotation and scale. These points are detected by a scale adapted Harris function and selected in scale-space by the Laplacian operator. The selected scale determines the size of the support region.

\section{$3.3 \quad$ SIFT}

The Scale-Invariant Feature Transform (SIFT) is an algorithm that detects distinctive keypoints from images and computes a descriptor for them. This algorithm was initially presented by Lowe [22] and used in object recognition tasks. The interest points extracted are said to be invariant to image scale, rotation, and partially invariant to changes in viewpoint and illumination. SIFT features are located at maxima and minima of a difference of Gaussians (DoG) function applied in scale space. They can be computed by building an image pyramid with resampling between each level [23]. In this work, we only use the detected points and we discard the descriptors.

\subsection{SURF}

Speeded Up Robust Features (SURF) is a scale and rotation invariant detector and descriptor which was recently presented by Bay et al. [24]. This detector is based on the Hessian matrix because of its accuracy and low computational time. SURF is based on sums of 2D Haar wavelet responses and makes an efficient use of integral images. According to [24], this algorithm outperforms existing methods with respect to repeatability, robustness and distinctiveness of the descriptors. As with SIFT features, we concentrate only on the detected points and we discard the descriptors. 


\subsection{SUSAN}

SUSAN (Smallest Univalue Segment Assimilating Nucleus) is an approach to low level image processing [25]. The SUSAN principle is implemented using digital approximation of circular masks. If the brightness of each pixel within a mask is compared with the brightness of that mask's nucleus, then an area of the mask can be defined which has the same brightness as the nucleus. SUSAN has been traditionally used for object recognition.

\section{Evaluation Methods}

To evaluate the previous methods we use sequences of images representing the same scene under different scales and viewpoints. In this section we explain how these sequences were evaluated. We first introduce the tracking method used to follow the interest points in each frame of the sequences. We then describe the measurements used to study the repeatability and robustness of each method under changes in scale an viewpoint. In this work we do not study the invariance under changes in illumination.

\subsection{Tracking}

For each image in a sequence, we first extract the interest points using the methods explained in Section 3. To track each point in successive images we try to match the interest points using the homography matrix for each pair of consecutive images as follows [26]. Given a point $Y$ in 3D space, we assume that this point projects at position $y_{1}=P_{1} Y$ in image $I_{1}$ and at position $y_{i}=P_{i} Y$ in image $I_{i}$, with projection matrices $P_{1}$ and $P_{i}$. If we suppose that the point $Y$ is detected in both images, then

$$
y_{i}=H_{1 i} \times y_{1} \text {, with } H_{1 i}=P_{i} P_{1}^{-1} \text {. }
$$

The homography matrix $H_{1 i}$ can be computed by selecting manually four correspondences of coplanar points between images 1 and $i$. Given a detected point in one image, we predict its position in the consecutive image using the homography matrix. If the predicted position lies at a distance below 2 pixels from an interest point detected in the second image, then we consider that the interest point is successfully tracked. If no interest point lies in the neighborhood of the predicted point, then the tracking of the point is lost. This method has been applied to sequences of images containing planar objects, since the computation of the homography matrix can only be made for coplanar points in the space. In the case of 3D images a similar method was used but with manual correction if the interest point in the second image was not found because of some occlusion.

An example of a tracking using this method is shown in Figure 1 in which the interest points were extracted with the Harris detector (white points). In this sequence, the red points in the last image indicate points that could be tracked along the whole sequence. The blue points are those ones that have been lost 


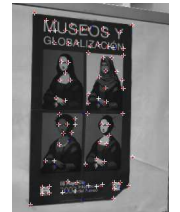

$0-1$

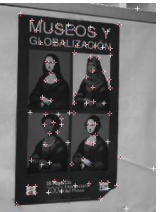

$1-2$

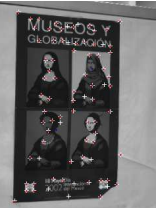

2-3

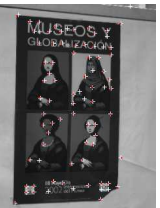

3-4

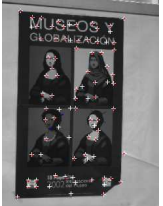

$4-5$

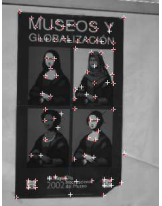

$5-6$

Fig. 1. Sequence of images with persistent points (red), lost points (blue) and points detected (white).

from the previous image. A point that is lost, even only once, is rejected by our tracking algorithm since we have considered that this kind of points are not stable enough for our purpose.

\subsection{Evaluation Measurements}

As explained in Section 3, we want to evaluate the detectors according to the SLAM requirements. In this sense, we have followed a repeatability criterion which means that the detection is independent of changes in the imaging conditions, i. e. scale and viewpoint. Applying our tracking method we first define the survival ratio $S_{i}$ in the frame $i$ as:

$$
S_{i}=\frac{\mathrm{np}_{i}}{\mathrm{np}_{0}} \cdot 100
$$

where $\mathrm{np}_{i}$ and $\mathrm{np}_{0}$ are the number of points detected in the frame $i$ and the first frame respectively. A perfect detector would detect the same points in the first and the last frame, i.e. $S_{i}=100 \%$ for every frame. However, as we will see in the experiments, we normally observe a decreasing tendency in $S_{i}$, meaning that some of the points observed in the first frame are lost in subsequent frames.

When the robot explores the environment, it is desirable to extract visual landmarks that are stable and can be detected in a number of $p$ consecutive frames $[17,10]$. As a result, the number of landmarks in the map is reduced and also the complexity of the SLAM problem. However, setting $p$ poses a problem: if $p$ is low, a high number of spurious points will be integrated in the map. If $p$ is high, the number of landmarks in the map will be too low. For example, when the robot turns, the landmarks disappear rapidly from the camera field of view and will not be integrated in the map if $p$ is high. Taking into account this requirement we analyze for how many frames we should track a landmark before integrating it in the map. We use the following conditional probability:

$$
P\left(t_{f_{a}} \mid t_{f_{b}}\right)=\frac{t_{f_{a}}}{t_{f_{b}}}
$$

where $t_{f_{i}}$ is the number of points tracked until frame $f_{i}$. This value represents the probability of an interest point to be tracked until frame $f_{a}$ given that it was 


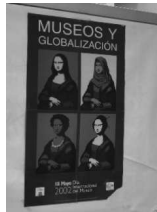

0

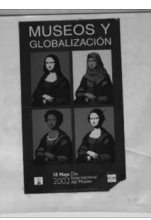

0

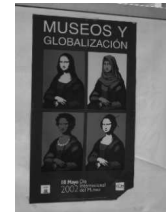

4

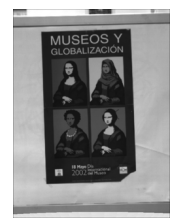

3

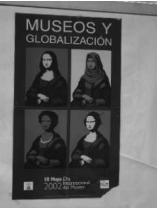

6

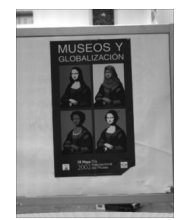

5

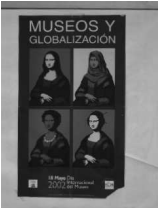

11

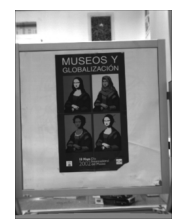

8

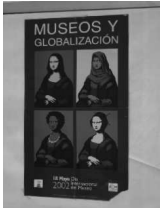

15

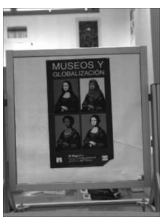

10

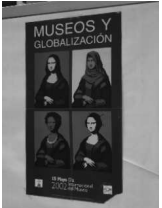

20

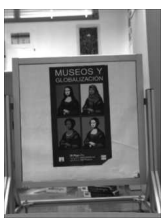

12

Fig. 2. The top sequence shows images of a poster from different viewpoints. The bootom sequence shows the same poster with changes in scale.

tracked until frame $f_{b}$. This value ranges between 0 and 1 . It is 0 when all points tracked until $f_{b}$ are lost in frame $f_{a}$, and 1 if both frames $f_{a}$ and $f_{b}$ contains the same tracked points.

Expression (5) gives a prediction of the survival of an interest point in future frames if the movement of the robot maintains similar. This expression can be used to estimate the number of frames $p$ a landmark has to be tracked before it is incorporated in the map.

\section{Experiments}

In order to evaluate the different interest point detectors, we captured 12 sequences of viewpoint changing images each containing 21 images. For each image we increased the angle in 2.5 degrees. Additionally we captured 14 sequences of images with scale changes each containing 12 images. In this last case the camera moved 0.1 meters in each image. The sequences contain images of planar objects (as posters) and 3D scenes. Examples of both types of images are shown in Figure 2 and Figure 3 respectively.

All images were captured using a STH-MDCS2 stereo head from Videre Design. Only one of the stereo images was used at each time to form the sequences. The stereo head was mounted on a robotic arm to achieve constant variations of viewing angle and distance change. Finally, the images were captured at different resolutions (320x240, 640x480 and 1280x960), so that the set of images could be as much representative as possible.

In a first experiment we analyze the repeatability of the different detectors in the sequences with changes in the viewpoint. In SLAM it is important that the landmarks detected with a certain angle and distance are also detected from 


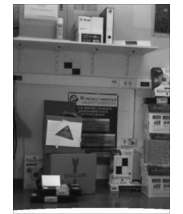

0

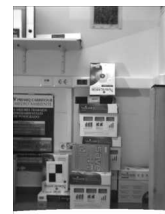

0

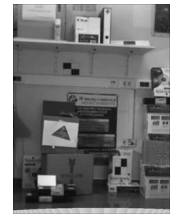

3

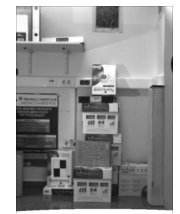

3

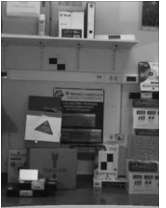

7

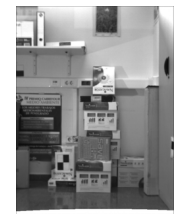

5

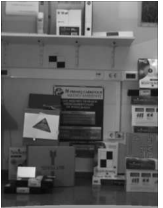

10

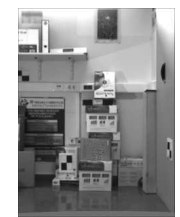

8

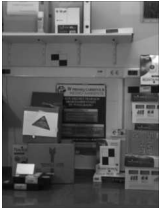

15

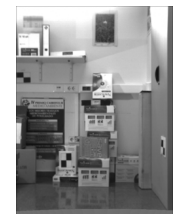

10

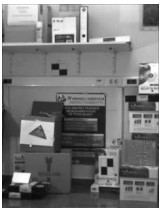

20

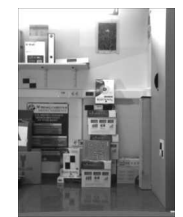

12

Fig. 3. The top sequence shows images of a $3 \mathrm{D}$ scene from different viewpoints. The bottom sequence shows a similar scene with changes in scale.

different ones. This comes from the fact that a mobile robot will see the same point in the scene from different poses in the environment. For this experiment we use as data all the sequences simultaneously and we calculate Expression (4) using the interest points of the images in all sequences, that is, $\mathrm{np}_{i}=\sum_{s=1}^{s=12} \mathrm{np}_{i}^{s}$ for all the 12 sequences.

As the left image of Figure 4 suggests, the Harris detector seems to be the most stable, being able to maintain almost $30 \%$ of the initial points in all images of the sequences with viewpoint changes of 50 degrees. Similar results are obtained when using Harris at different scales (right image of Figure 4). The SIFT detector obtain also good results at different viewpoints, but it gets worse under changes in scale.

Figure 5 presents a different way of comparing the detectors. In this case, the plots show the probability that a point is found in the last frame given that it was tracked until the frame $i$, as shown in Expression (5). Again the Harris detector gives the best results under changes in viewpoint and scale. We can see that, for example, a Harris-point which is tracked for 10 frames will have a probability of 0.7 of being tracked until frame 20 .

Although the plots of Figure 4 and Figure 5 contain similar information, the second one can be used to further discriminate between different detectors. For example, in the right image of Figure 4, the SIFT, SURF and Harris-Laplace descriptors show a similar behavior, however the right image in Figure 5 shows that the SURF descriptor is more stable. If we follow a landmark extracted with the SURF descriptor for 6 frames, it will have a probability of 0.5 of being tracked until frame 12 , while this probability decreases to 0.4 when the point was extracted using SIFT or Harris-Laplace. 

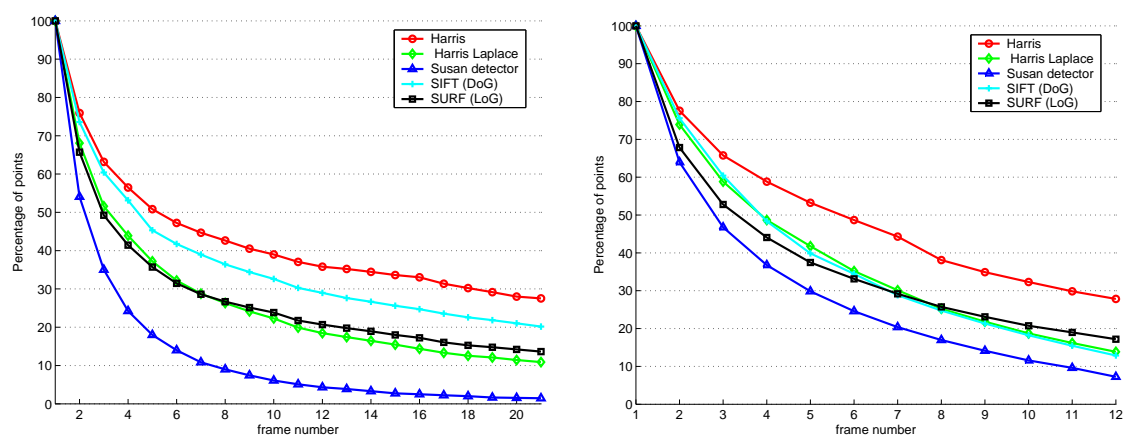

Fig. 4. The left plot shows the survival ratio for each of the frames in the sequences with change in viewpoint. The right plot shows the same value in the sequences with change in scale.
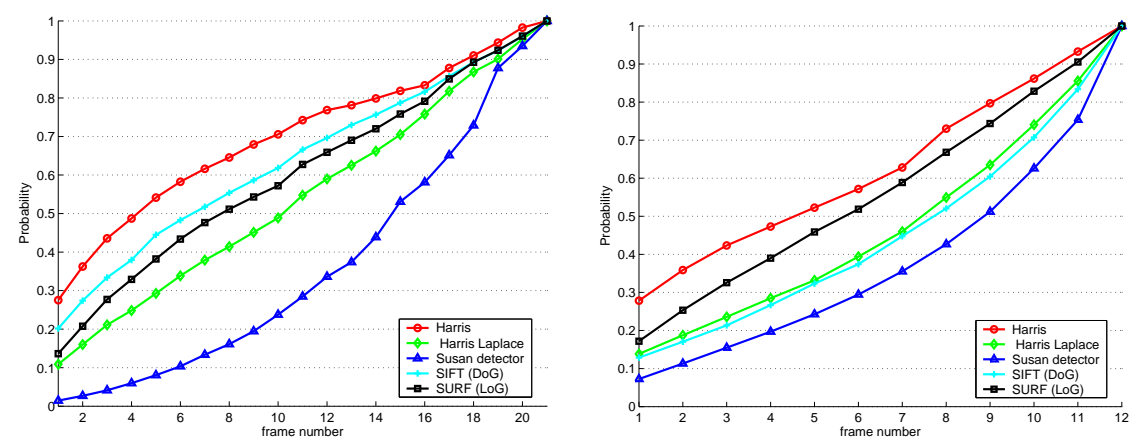

Fig. 5. The figures show the probability of a point being detected in the last frame given that it was detected in the frame $i$ of the sequences: left with changes in viewpoint, and right with changes in scale.

Table 1 presents the number of interest points detected in the first image and the number of points that were tracked until the last frame. It can be clearly seen that the number of points detected differs when using different methods. This stems from the fact that we are using an heterogeneous image database and it is not possible to adjust each of the detectors in a way that the number of detected points is the same for all the methods. For instance, the parameters for each of the five methods can be adjusted in a way that the number of points detected in a single image would be equal. However, the same parameters applied to a different image would result in differing number of points detected. In consequence, the results presented here are normalized to the number of points that appear in the first frame, so that they can be compared. 
Table 1. Number of points detected in the first and last image of each sequence

\begin{tabular}{|l|c|c|c|c|c|}
\hline Changes in Viewpoint & Harris & Harris Laplace & SUSAN & SIFT & SURF \\
\hline Number of points detected in the first image & 2064 & 2588 & 2967 & 3808 & 10372 \\
\hline Number of points tracked to the last image & 568 & 282 & 68 & 407 & 1415 \\
\hline Changes in Scale & Harris & Harris Laplace & SUSAN & SIFT & SURF \\
\hline Number of points detected in the first image & 5728 & 5685 & 6421 & 8207 & 24996 \\
\hline Number of points tracked to the last image & 1594 & 788 & 465 & 1058 & 4295 \\
\hline
\end{tabular}

\section{Conclusions}

In this paper we presented an evaluation of different interest point detectors. We focused on the use of interest points in visual-based SLAM. For this purpose we analyzed each detector according to the properties desired for visual landmarks: repeatability and accuracy. The results of the experiments showed the behavior of five different detectors under changes in viewpoint and scale. We believe that this information will be usefull when selecting an interest point detector as visual landmark extractor for SLAM.

\section{Acknowledgment}

This work has been supported by the Spanish Government under the projects DPI2004-07433-C02-01 and PCT-G54016977-2005.

\section{References}

1. Grisetti, G., Stachniss, C., Burgard, W.: Improved techniques for grid mapping with rao-blackwellized particle filters. IEEE Transactions on Robotics 23(1) (2007)

2. Hähnel, D., Burgard, W., Fox, D., Thrun, S.: An efficient FastSLAM algorithm for generating maps of large-scale cyclic environments from raw laser range measurements. In: IEEE/RSJ Int. Conf. on Intelligent Robots \& Systems, Las Vegas, NV, USA (2003)

3. Leonard, J., Durrant-Whyte, H.: Mobile robot localization by tracking geometric beacons. IEEE Transactions on Robotics and Automation 7(4) (1991)

4. Biber, P., Andreasson, H., Duckett, T., Schilling, A.: 3d modelling of indoor environments by a mobile robot with a laser scanner and panoramic camera. In: IEEE/RSJ Int. Conf. on Intelligent Robots \& Systems. (2004)

5. Eustice, R., Singh, H., Leonard, J.: Exactly sparse delayed-state filters. In: IEEE Int. Conf. on Robotics \& Automation. (2005)

6. Triebel, R., Burgard, W.: Improving simultaneous mapping and localization in 3d using global constraints. In: National Conference on Artificial Intelligence. (2005)

7. Lemaire, T., Lacroix, S.: Monocular-vision based SLAM using line segments. In: IEEE Int. Conf. on Robotics \& Automation. (2007)

8. Frintrop, S., Jensfelt, P., Christensen, H.I.: Attentional landmark selection for visual slam. In: IEEE/RSJ Int. Conf. on Intelligent Robots and Systems. (2006) 
9. Little, J., Se, S., Lowe, D.: Vision-based mobile robot localization and mapping using scale-invariant features. In: IEEE Int. Conf. on Robotics \& Automation. (2001)

10. Gil, A., Reinoso, O., Burgard, W., Stachniss, C., Martínez Mozos, O.: Improving data association in rao-blackwellized visual SLAM. In: IEEE/RSJ Int. Conf. on Intelligent Robots \& Systems. (2006)

11. Valls Miro, J., Zhou, W., Dissanayake, G.: Towards vision based navigation in large indoor environments. In: IEEE/RSJ Int. Conf. on Intelligent Robots \& Systems. (2006)

12. Davison, A.J., Murray, D.W.: Simultaneous localisation and map-building using active vision. IEEE Transactions on Pattern Analysis and Machine Intelligence (2002)

13. Hygounenc, E., Jung, I.K., Souères, P., Lacroix, S.: The autonomous blimp project of laas-cnrs: Achievements in flight control and terrain mapping. International Journal of Robotics Research 23(4-5) (2004)

14. Murillo, A.C., Guerrero, J.J., Sagüés, C.: Surf features for efficient robot localization with omnidirectional images. In: IEEE Int. Conf. on Robotics \& Automation. (2007)

15. Jensfelt, P., Kragic, D., Folkesson, J., Björkman, M.: A framework for vision based bearing only 3D SLAM. In: IEEE Int. Conf. on Robotics \& Automation. (2006)

16. Se, S., Lowe, D.G., Little, J.: Vision-based mobile robot localization and mapping using scale-invariant features. In: IEEE Int. Conf. on Robotics \& Automation. (2001)

17. Little, J., Se, S., Lowe, D.: Global localization using distinctive visual features. In: IEEE/RSJ Int. Conf. on Intelligent Robots \& Systems. (2002)

18. Mikolajczyk, K., Schmid, C.: A performance evaluation of local descriptors. IEEE Transactions on Pattern Analysis and Machine Intelligence 27(10) (2005)

19. Schmid, C., Mohr, R., Bauckhage, C.: Evaluaton of interest point detectors. International Journal of computer Vision 37(2) (2000)

20. Harris, C.G., Stephens, M.: A combined corner and edge detector. In: Alvey Vision Conference. (1998)

21. Mikolajczyk, K., Schmid, C.: Indexing based on scale invariant interest points. In: Int. Conf. on Computer Vision. (2001)

22. Lowe, D.: Object recognition from local scale-invariant features. In: Int. Conf. on Computer Vision. (1999)

23. Lowe, D.: Distinctive image features from scale-invariant keypoints. Int. Journal of computer Vision 2(60) (2004)

24. Bay, H., Tuytelaars, T., Van Gool, L.: Object recognition from local scale-invariant features. In: European Conference on Computer Vision. (2006)

25. Smith, S.: A new class of corner finder. In: British Machine Vision Conference. (1992)

26. Dork, G., Schmid, C.: Selection of scale invariant neighborhoods for object class recognition. In: Int. Conf. on Computer Vision. (2003) 